Supporting Information

\title{
Optical Properties and Applications of Photonic
}

\section{Shells}

Dan-Bi Myung and Soo-Young Park*

Department of Polymer Science \& Engineering, Polymeric Nanomaterials Laboratory,

School of Applied Chemical Engineering, Kyungpook National University, 80 Daehak-ro,

Buk-gu, 41566 Daegu, Korea

*E-mail: psy@knu.ac.kr 
Preparation of CLC shells and description of microfluidics set-up: The outer diameter of the round capillaries $(0.87 \mathrm{~mm})$ was comparable to the inner diameter of the square capillary $(0.9 \mathrm{~mm})$, such that a round capillary could be inserted into the square capillary from each end to achieve a tight-fitting, coaxial alignment. The tapered ends of the two capillaries were adjacent to each other at the center of the square capillary, as shown in Figure S7a. The diameters of the left and right tapered ends were $110 \pm 10 \mu \mathrm{m}$ and $250 \pm 20 \mu \mathrm{m}$, respectively. For the preparation of the CLC shells, the inner aqueous phase was allowed to flow through the left capillary, while the CLC flowed in the same direction in the square capillary; the outer continuous aqueous phase flowed in the opposite direction. The density of the aqueous phase was controlled by mixing glycerol with water to make a CLC shell with a uniform thickness by matching the density of the aqueous phase with that of the CLC mixture. Details relating to the mixing ratio will be discussed in the results and discussion section. Droplets of the inner aqueous phase formed within the CLC phase as the Rayleigh instability broke up the streams of the two co-flowing fluids. The CLC and continuous aqueous phases met between the ends of the two round capillaries, and their immiscibility caused the resulting shells to collect in the right-hand capillary. Flow rates were controlled using a pneumatic microfluidic flow-rate control system (OB1 pressure controller, Elveflow, France), which is capable of pumping three fluids at a specified velocity. This system was connected to the microcapillary device using shrinkable connector tubes and flexible plastic tubing (Norton, $0.51 \mathrm{~mm}$ inner diameter, $1.52 \mathrm{~mm}$ outer diameter). By pumping air at a finely controlled rate into the vials containing the liquids, the Elveflow unit pressurized the vials, which caused the fluids to flow through the tubes and into the device. In order to stabilize the shells and prevent them from rupturing, $1 \mathrm{wt} \%$ PVA was added to the outer aqueous phase, which 
adsorbs at the CLC/aqueous interface and ensures planar orientation. The left round capillary was coated with tetraethylorthosilicate (TEOS) to increase the wettability of the CLC mixture on the glass surface. The contact angle of water on the TEOS-coated glass was $13.3^{\circ}$, which is lower than $28.7^{\circ}$ from the bare glass, indicating that the wettability is improved using TEOS coating. Square and right round capillaries were used without any coatings. TEOS coating was performed on an $\mathrm{O}_{2}$ plasma-treated glass capillary. The TEOS-coated glass capillary was washed with flowing DI water to remove excess material. 


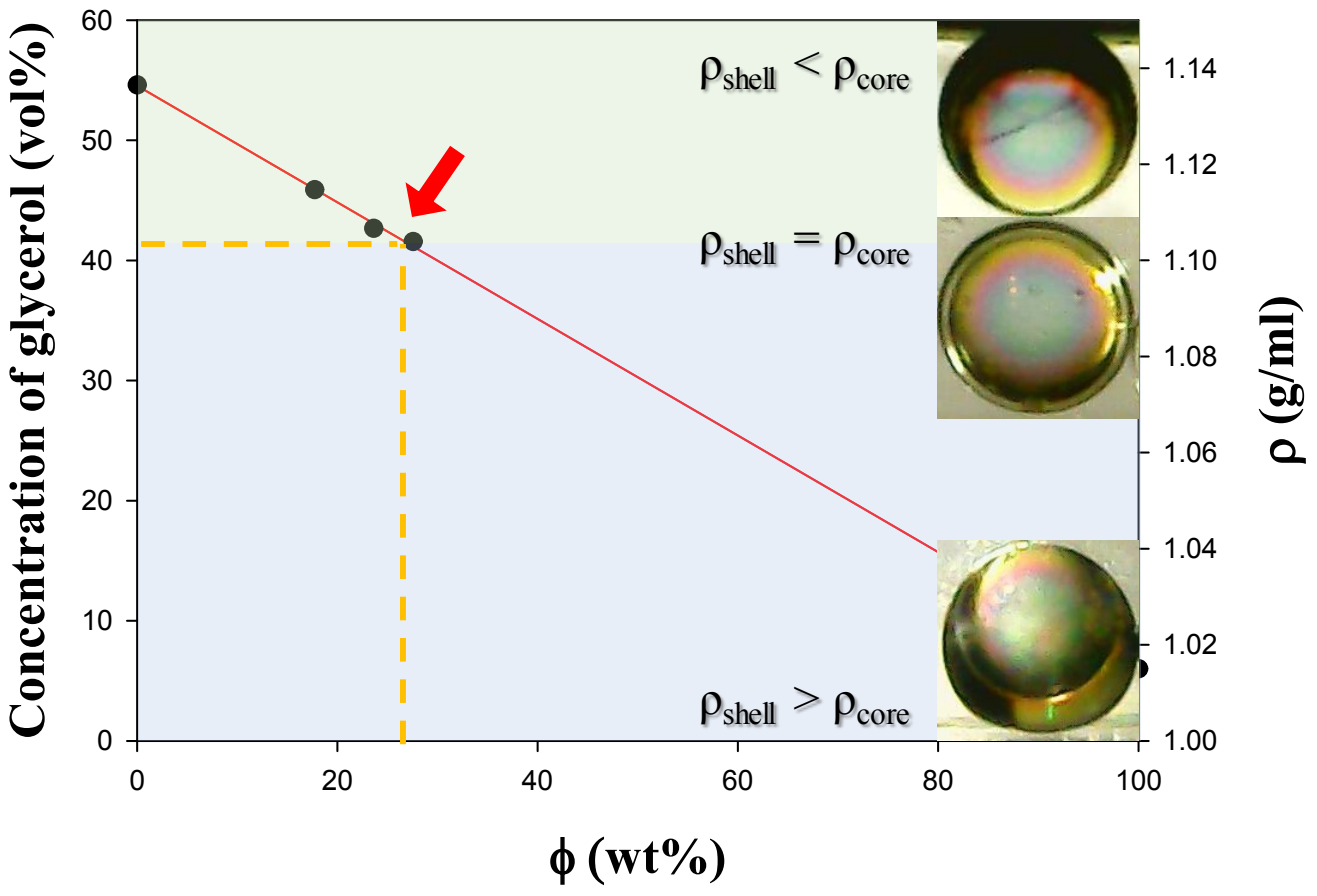

Figure S1. Density of the CLC shells as a function of $\phi$, which was measured using a floating method with a mixture of glycerol and water. 
(i)

(ii)

(iii)

(iv)

(a)

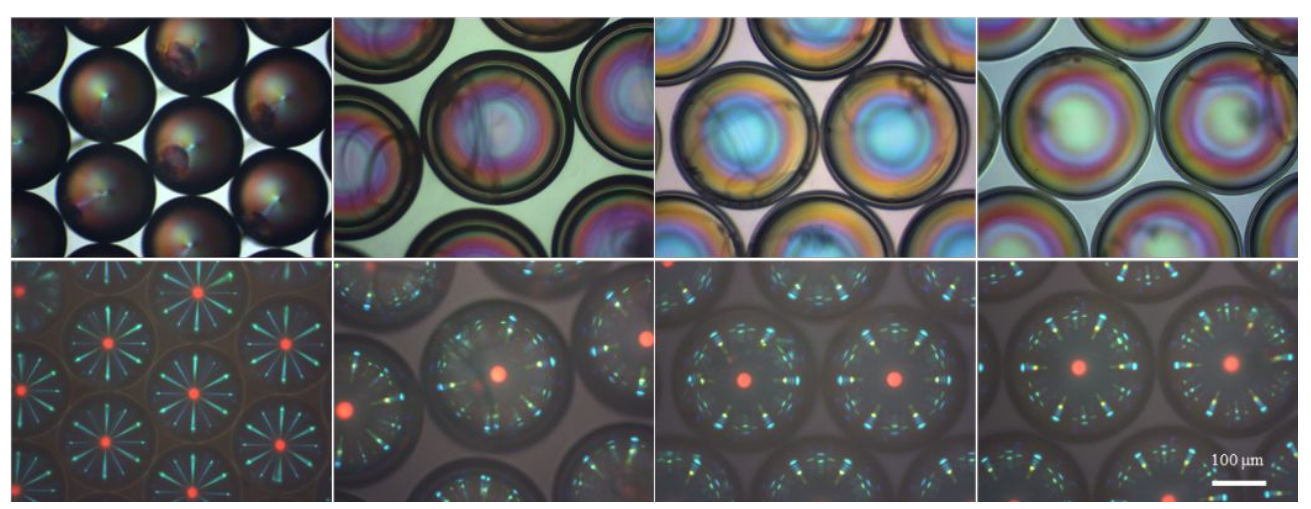

Figure S2. (a) TOM and (b) ROM images of the CLC (i) single droplet and (ii, iii, iv) shells in water prepared at $\varphi=42$ vol\% with shell thicknesses of (ii) 28 , (iii) 16 , and (iv) $8 \mu \mathrm{m}$. 
(a)

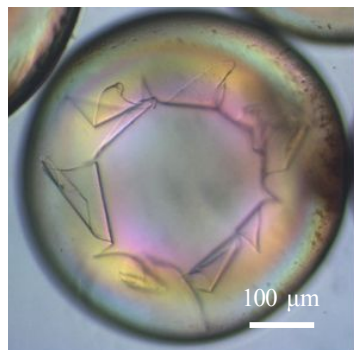

(b)

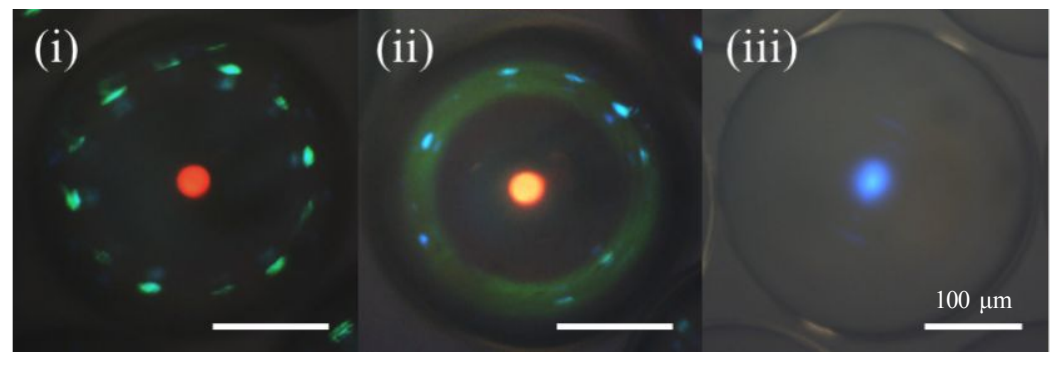

Figure S3. ROM images of the $\mathrm{CLC}_{\text {solid }}$ shells in water prepared (a) at $\varphi=29 \mathrm{vol} \%$ after UV curing and CB15 extraction, resulting in the fractured hole at the center, and at $\varphi=$ (b) 42 vol\% (i) before and after (ii) UV curing and (iii) CB15 extraction; $(r, \Delta r)=(155,13),(154$, 12), and $(165,7) \mu \mathrm{m}$ for (i), (ii), and (iii), respectively. 
(i)

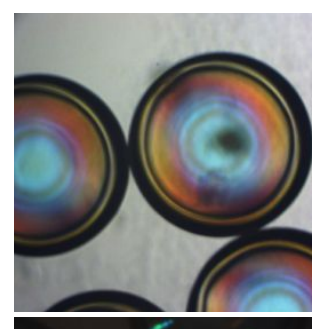

(a)

(b)
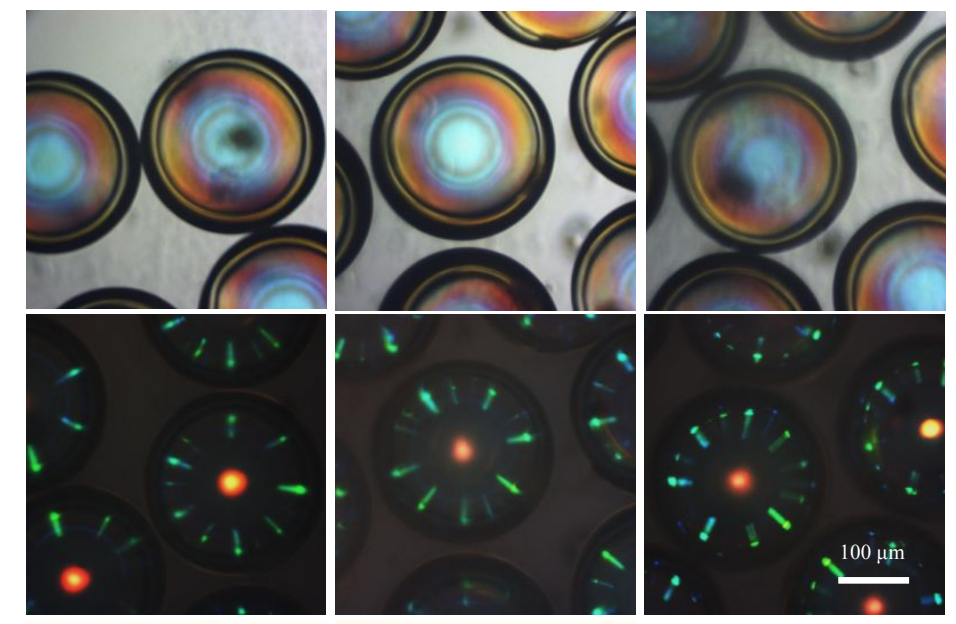

(c)

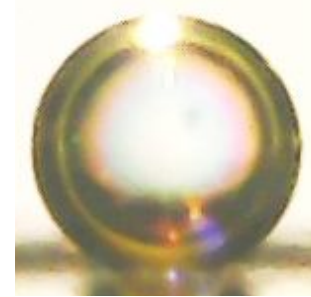

(ii)

(iii)

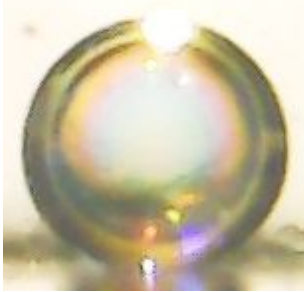

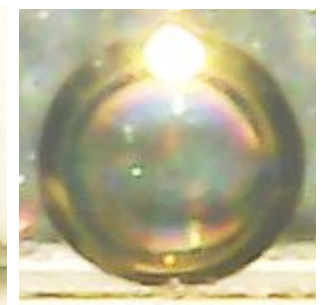

Figure S4. (a, c) TOM and (b) ROM images of the (a, b) top and (c) side views of the $\mathrm{CLC}_{\text {solid }}$ shells ( $\phi=26 \mathrm{wt} \%$ and $\varphi=42 \mathrm{vol} \%$ ) which were UV cured for 10 min with relative intensities of (i) 33 , (ii) 80 , and (iii) $133 \mu \mathrm{W} / \mathrm{cm}^{2}$. 
(a)

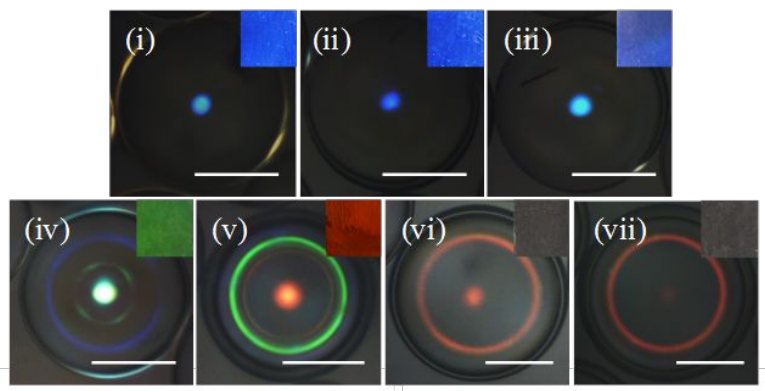

(b)

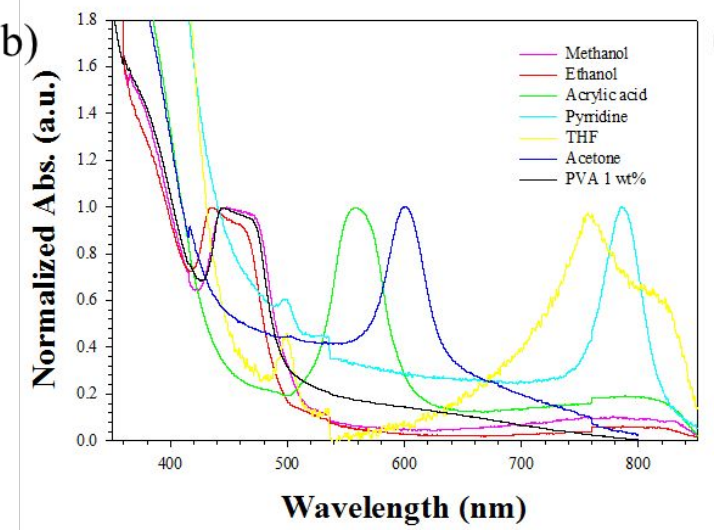

(c)

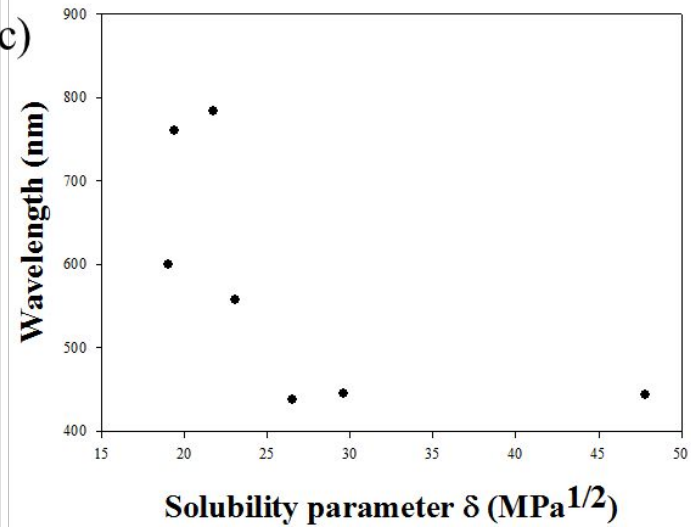

Figure S5. ROM images of the $\mathrm{CLC}_{\text {solid }}$ shells ( $\varphi=42$ vol\%) in (i) water, (ii) ethanol, (iii) methanol, (iv) acrylic acid, (v) acetone, and (vi) pyridine; insets are their images from film samples, (b) UV-vis spectra of the CLC films (a, inset), and (c) the $\lambda_{\mathrm{PBG}}$ from (b) as a function of the solubility parameter; $(r, \Delta r)=(101,6),(102,7),(101,7),(102,8),(106,10)$, $(124,16)$, and $(126,18) \mu \mathrm{m}$ for (i), (ii), (iii), (iv), (v), (vi) and (vii), respectively. 


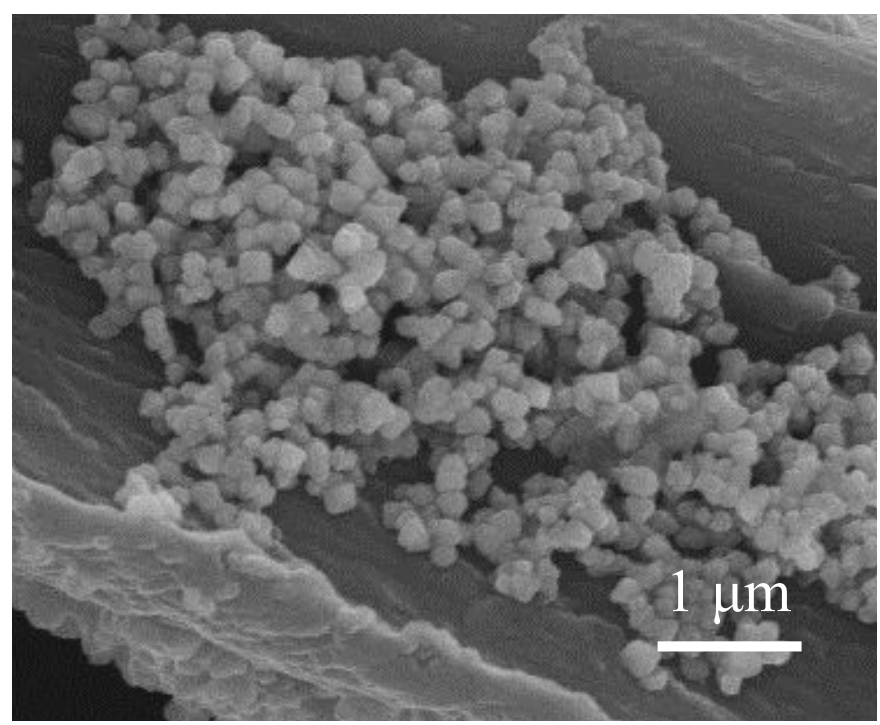

Figure S6. SEM micrograph of the $\mathrm{Fe}_{3} \mathrm{O}_{4}$ nanoparticles with a diameter of $150 \mathrm{~nm}$ inside of the $\mathrm{CLC}_{\text {solid }}$ shells (prepared at $\varphi=42 \mathrm{vol} \%$ ); the sample was fractured by finger pressure. 
(a)

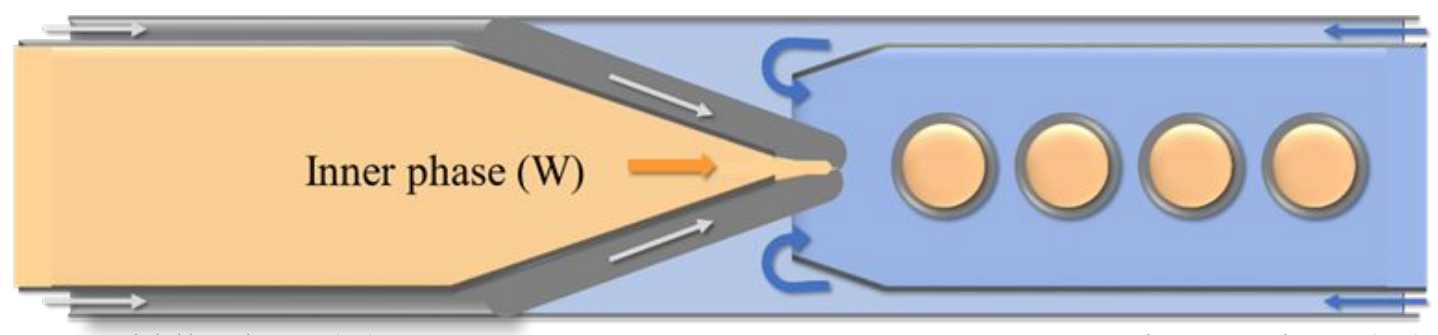

Middle phase (O)

Continuous phase (W)

(b)

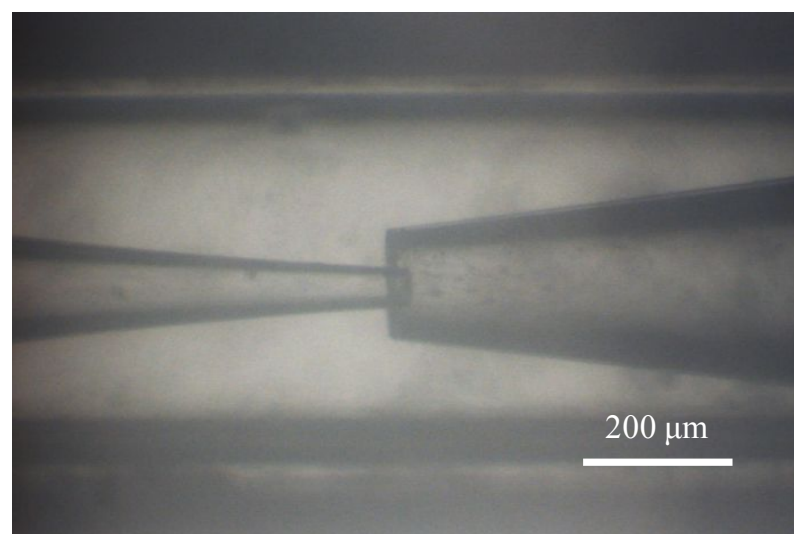

Figure S7. (a) Schematic and (b) photograph of the microfluidic setup for producing monodisperse CLC shells using combined co-flow and flow-focusing geometry in the glass capillary chip. 